\title{
Attenuation coefficient as a quantitative parameter for analyzing cataracts with optical coherence tomography
}

\author{
Pablo Eugui ${ }^{1, *}$, Danielle J. Harper ${ }^{1}$, Johanna Gesperger ${ }^{1,2}$, Antonia Lichtenegger ${ }^{1}$, Conrad W. Merkle ${ }^{1}$, Martin Glösmann ${ }^{3}$ \\ and Bernhard Baumann ${ }^{1}$ \\ ${ }^{1}$ Center for Medical Physics and Biomedical Engineering, Medical University of Vienna, Austria \\ ${ }^{2}$ Division of Neuropathology and Neurochemistry, Department of Neurology, Medical University of Vienna, Austria \\ ${ }^{3}$ Core Facility for Research and Technology, University of Veterinary Medicine, Austria
}

\begin{abstract}
Crystalline lenses of mice were imaged in vivo with a custom-made swept-source optical coherence tomography system. The use of the attenuation coefficient as a quantitative parameter for investigating the lens opacities magnitude is proposed, demonstrating a significant difference between the values retrieved from cataractous and normal mouse lenses.
\end{abstract}

\section{Introduction}

Cataracts are the major cause of blindness worldwide, they are characterized by a progressive loss of transparency of the ocular lens which ultimately leads to poor vision [1]. Optical coherence tomography (OCT) has been proposed as a non-invasive imaging technique to visualize and quantify the morphology of these opacifications in human patients and animal models $[2,3]$. In biomedical applications, the attenuation coefficient of the tissue recovered by optical methods has been used as a parameter for classification and diagnosis [4]. Previously we presented OCT as a tool to visualize different opacifications in vivo in the crystalline lens of mice [3]. Here we want to extend our previous results with the use of the optical attenuation coefficient as a further measure to analyze and characterize cataract formations.

\section{Methods}

In this paper we used the custom-made OCT system illustrated in Fig. 1(A), which operates at a central wavelength of $1310 \mathrm{~nm}$ and provides an axial resolution of $\sim 6.5 \mu \mathrm{m}$ in air with a sensitivity of $100 \mathrm{~dB}$. The OCT device was described in detail elsewhere along with the experimental procedure of the data used for this work [3]. Data sets with $400 \times 400 \times 768$ pixels $(3 \times 3 \times 5 \mathrm{~mm})$ were acquired in the mouse anterior segment, repeating 4 scans per position to increase the signal-to-noise ratio. A post-processing pipeline was implemented in MATLAB (MATLAB, R2015b, MathWorks). Every acquired spectrum was aligned with a unique spectrum using crosscorrelation to reduce shifts induced by A-line trigger jitter. Background removal and spectral shaping were then performed before averaging the resulting repeated scans after Fourier transformation. The lens surface was segmented and the equation

$$
y=A \cdot \exp (-\mu z)
$$

was fitted to the intensity profiles in all A-scans for a range spanning $0.5 \mathrm{~mm}$ in depth underneath the surface. In the fit, the scaling factor $A$ and the attenuation coefficient $\mu$ were the free running parameters to be optimized by the algorithm. En-face attenuation maps were then calculated. A similar region of interest consisting of $0.16 \mathrm{~mm}^{2}$ was analyzed for cataract and noncataract eyes and compared as shown in Figure 1(B). A Student's t-test was used to analyze the difference between the average attenuation coefficients of the two groups.

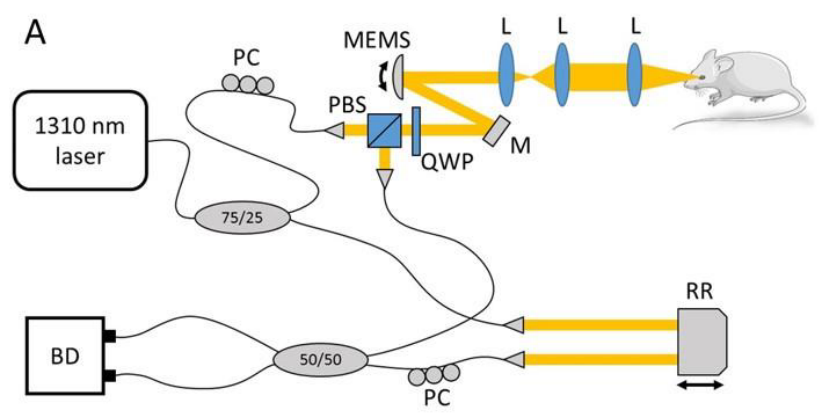

B

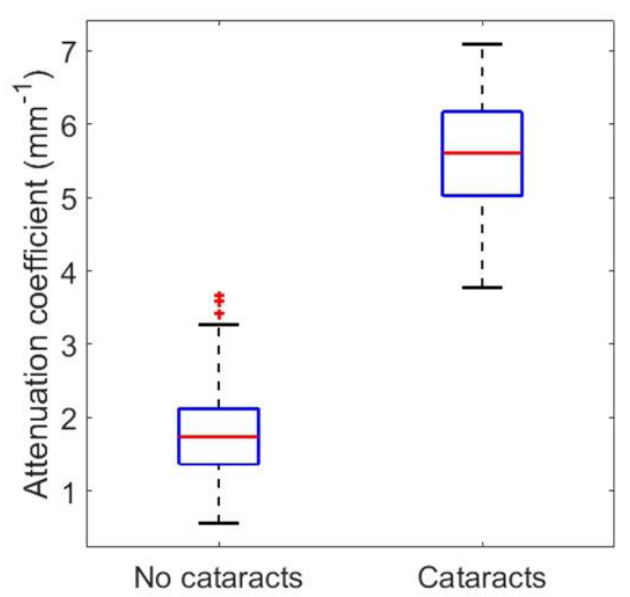

Fig. 1. A) Home-built OCT device used for mouse lens imaging. PBS: beam splitter, BD: balanced detector, L: lens, M: mirror, MEMS: microelectromechanical scanner, PC: polarization controller, QWP: quarter wave plate, RR: retroreflector. B) Average attenuation coefficient compared between normal and cataract eyes.

\footnotetext{
* Corresponding author: pablo.euguiarrizabalaga@meduniwien.ac.at
} 

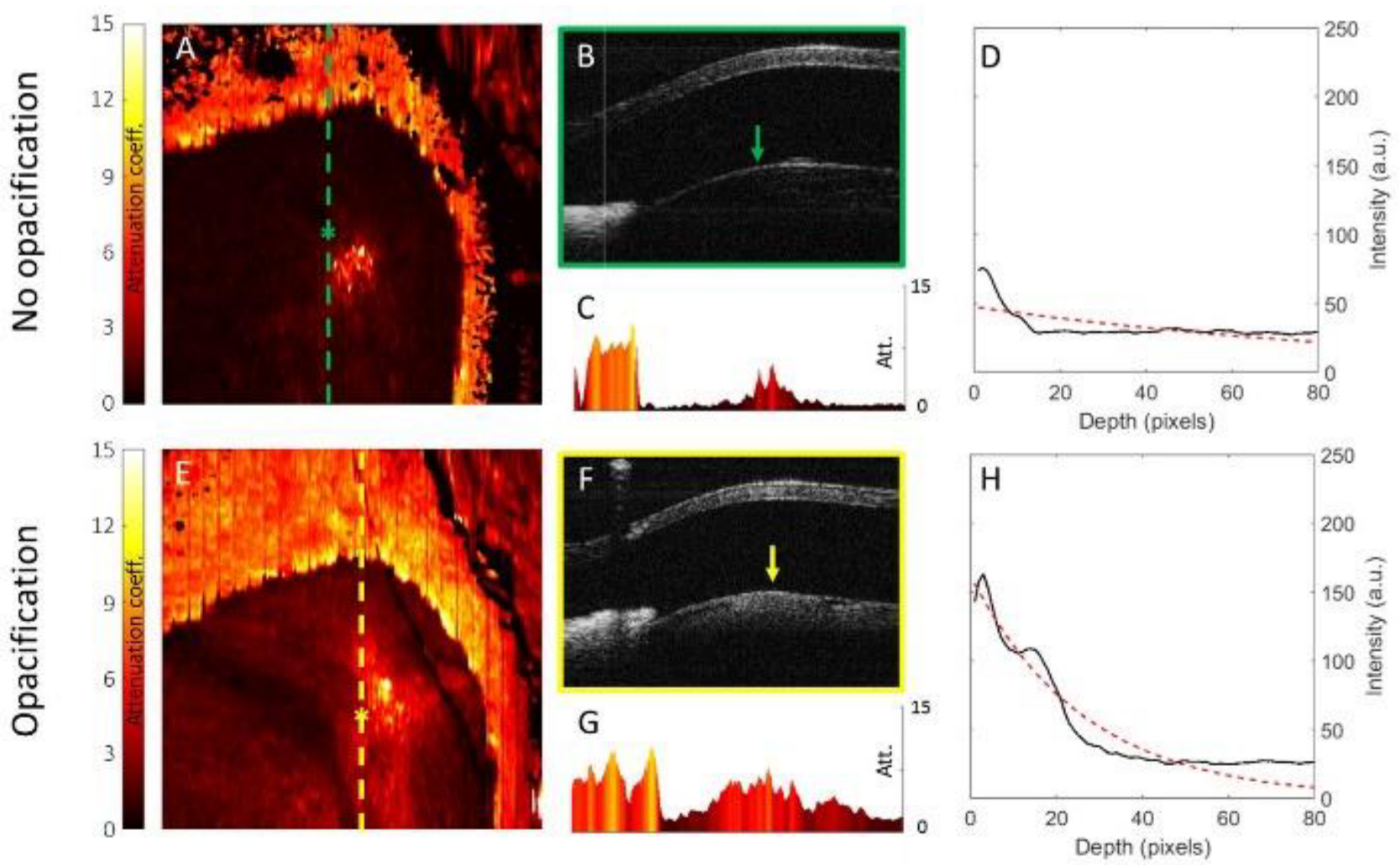

Fig. 2. A) Attenuation map of a lens without opacification. Aside from the high values caused by a strong surface reflection in the center at the anterior lens pole, only low attenuation is observed in the lenticular area. B) When looking at an individual B-scan marked with a yellow line in A), only the lens surface is visible without showing any scattering underneath. C) Attenuation coefficient profile of the lens in the B-scan shown in B). Low values can be seen in the lens except the central reflection area and those values corresponding to the iris on the left. D) A-scan profile and exponential fit of the pixels selected underneath the lens surface in the location indicated by the green arrow in B). E) Attenuation map of a lens with an opacity present in the lens cortex. Higher attenuation coefficients can be observed in the lens. F) Cross-section of B-scan located at the position indicated by the yellow line in E). A cloudy opacity can be seen underneath the lens surface. G) Attenuation profile of the lens in the B-scan shown in F) where higher values can be observed in the lens. H) Depth profile and exponential fit of the selection underneath the lens indicated by the yellow arrow in F).

\section{Results}

An attenuation map of a mouse eye without cataract is shown in Figure 1(A). In the eyes where no opacification was observed, only a very subtle scattering signal was detected in the lens due to its transparency, which led to low attenuation coefficients as shown in the Figure 1(B) and 2(ACD). An intensity profile of a lens without opacification is shown in Figure 2(D). When an opacity was present in the mouse eye, the attenuation within the lens was stronger as shown in Figure 1(E). This was due to a cloudy hyperscattering signal caused by the cataract formed in the lens cortex as shown in Figure 2(F). In the lateral attenuation profile of a B-scan with opacification, higher values can be observed in addition to the ones corresponding to the iris on the left as shown in Figure $2(\mathrm{G})$. This is also visible in the axial intensity profile shown in Figure 2(H) where higher values, rising as well as a steeper slope of the fitted exponential can be observed. When comparing similar regions between cataractous and non cataractous eyes, a higher attenuation $\left(5.5 \pm 0.7 \mathrm{~mm}^{-1}\right)$ was found in cataractous areas compared to the non-cataracts regions $\left(1.8 \pm 0.5 \mathrm{~mm}^{-1}\right)$, showing a significant difference.

\section{Conclusion}

A custom-made OCT system was used for volumetric in vivo visualization of the crystalline lens in mice. A postprocessing pipeline was implemented to obtain the attenuation coefficient, which revealed a significant difference between cataracts and normal eyes.

This research was funded by the European Research Council (ERC StG 640396 OPTIMALZ).

\section{References}

1. Thylefors, B. Int. Ophthalmol. 14(3) 211-219 (1990)

2. de Castro, A, et al. Invest. Ophthalmol. Vis. Sci. 59(2) 897-903 (2018)

3. Eugui, P. et al. Biomed. Opt. Express. 11(4) 20852097 (2020)

4. Chan, S. et al. J. Biomed. Opt. 24(9), 090901 (2019)

\footnotetext{
Corresponding author: pablo.euguiarrizabalaga@meduniwien.ac.at
} 